\title{
Small Fluorescein Arsenical Hairpin-Based Förster Resonance Energy Transfer Analysis Reveals Changes in Amino- to Carboxyl-Terminal Interactions upon OAG Activation of Classical Transient Receptor Potential 6 $\mathbf{s}$
}

\author{
Susanne Fiedler, Ursula Storch, Serap Erdogmus, Thomas Gudermann, \\ Michael Mederos y Schnitzler, and (1)Alexander Dietrich \\ Walther-Straub-Institute of Pharmacology and Toxicology, Member of the German Center for Lung Research (DZL) (S.F., U.S., \\ S.E., T.G., M.M.S., A.D.) and Institute for Cardiovascular Prevention (IPEK) (U.S.), Ludwig-Maximilians-Universitaet Muenchen, \\ Munich, Germany; German Centre for Cardiovascular Research (DZHK), Munich Heart Alliance, Munich, Germany (T.G., M.M.S.); \\ and Comprehensive Pneumology Center Munich (CPC-M), Munich, Germany (T.G., A.D.)
}

Received January 24, 2019; accepted May 2, 2019

\begin{abstract}
Although the overall structure of many classical transient receptor potential proteins (TRPC), including human and murine TRPC6, were recently resolved by cryoelectron microscopy analysis, structural changes during channel activation by 1-oleoyl-1-acetyl-sn-glycerol (OAG), the membrane-permeable analog of diacylglycerol, were not defined. Moreover, data on carboxyl- and amino-terminal interactions were not provided, as the amino-terminal regions of murine and human TRPC6 were not resolved. Therefore, we employed a Förster resonance energy transfer (FRET) approach using a small fluorescein arsenical hairpin (FIAsH) targeted to a short tetracysteine sequence at the unresolved amino-terminus and cerulean, a cyan fluorescent protein, as a tag at the carboxyl-terminus of the murine TRPC6 protein. After OAG as well as GSK-1702934A activation, FRET efficiency was simultaneously and significantly reduced, indicating a decreased interaction between the amino
\end{abstract}

to carboxyl termini in the functional tagged murine TRPC6 tetramer (TRPC6 WT) heterologously expressed in human embryonic kidney 293T cells. There was a significant reduction in the FRET signal obtained from analysis of murine TRPC6 FRET constructs with homologous amino-terminal mutations (M131T, G108S) that had been identified in human patients with inherited focal segmental glomerulosclerosis, a condition that can lead to end-stage renal disease. A novel, designed loss-of-function TRPC6 mutation (N109A) in the amino-terminus in close proximity to the carboxyl-terminus produced similar FRET ratios.

\section{SIGNIFICANCE STATEMENT}

Our data show for the first time that FIAsH-tagging of ion channels is a promising tool to study conformational changes after channel opening and may significantly advance the analysis of ion channel activation as well as their mutants involved in channelopathies.

\section{Introduction}

Transient receptor potential (TRP) channels represent a diverse protein superfamily of 28 members known as versatile cellular sensors and effectors. Among the six TRP families, the classical transient receptor potentials (TRPCs) were the first cloned mammalian TRP channels. Members of this family share the highest homology to the initially identified channels in the eyes of the fruit fly Drosophila melanogaster by characterization of a mutant with a short-lived depolarizing

This work was supported by the Deutsche Forschungsgemeinschaft [TRR152, project 16] to S.F. and A.D. and [Grant 406028471] to U.S. The authors declare that there are no conflicts of interest regarding the publication of this paper.

https://doi.org/10.1124/mol.119.115949.

S This article has supplemental material available at molpharm.aspetjournals.org current termed transient receptor potential. All TRPC family members harbor an invariant sequence, the TRP box (containing the amino acid sequence: EWKFAR) in its carboxyl-terminal tail as well as ankyrin repeats in the amino-terminus. They are composed of intracellular amino and carboxyl termini, six membrane-spanning helices (S1S6), and a presumed pore-forming loop between S5 and S6 [reviewed in Dietrich et al. (2005b)]. For a functional TRPC ion channel complex, four monomers of the same type in a homotetrameric complex or four different TRPC monomers forming a heterotetrameric channel are essential. All TRPC channels except TRPC1, whose role as ion channel or channel regulator is still a matter of debate (Dietrich et al., 2014), share a common activator, diacylglycerol (Dietrich et al., 2005b; Storch et al., 2017), and are involved in complex cellular signal transduction cascades.

ABBREVIATIONS: BTDM, 3-(1,3-benzodioxol-5-yl)-1-phenylprop-2-en-1-one; DMSO, dimethyl sulfoxide; FIAsH, fluorescein arsenical hairpin; FRET, Förster resonance energy transfer; FSGS, focal segmental glomerulosclerosis; GOF, gain of function; GPCR, G protein-coupled receptors; GSK-170, GSK-1702934A; HBSS, Hanks' balanced salt solution; LOF, loss of function; OAG, 1-oleoyl-1-acetyl-sn-glycerol; TRP, transient receptor potential; TRPC, classical transient receptor potential; WT, wild-type. 
Diacylglycerol is produced from phosphatidylinositol 4,5-bisphosphate by phospholipase C isozymes activated after agonist binding to appropriate receptors [reviewed in Dietrich et al. (2005a)].

The sixth member of the TRPC family, TRPC6, was originally cloned from mouse brain (Boulay et al., 1997) and human placenta (Hofmann et al., 1999) and is predominantly expressed in lung. In pulmonary smooth muscle cells TRPC6 is essential for acute hypoxic vasoconstriction (Weissmann et al., 2006) and regulates induction of endothelial permeability by lung edema during ischemia-reperfusion injury (Weissmann et al., 2012). Other functions, especially in the kidney, immune and blood cells, as well as in the brain, still need to be explored (reviewed in Dietrich and Gudermann (2014)).

Activating so called "gain of function (GOF)" as well as inactivating "loss of function (LOF)" mutants of TRPC6 were identified in the kidney glomeruli of patients with proteinuria resulting from inherited forms of focal segmental glomerulosclerosis (FSGS) (Reiser et al., 2005; Winn et al., 2005; Mottl et al., 2013; Riehle et al., 2016). The glomerular filter is composed of a fenestrated capillary endothelium, the glomerular basement membrane, and podocyte foot processes that are connected by a structure known as the slit diaphragm. In recent years, podocytes have emerged as central players in glomerular research, because podocyte damage weakens the function of the filter and leads to proteinuria, a characteristic feature of most glomerular diseases. For this reason ion channels expressed in glomerular podocytes and their foot processes have attracted special attention, and it was hypothesized that the identified TRPC6 mutations may cause FSGS by inducing podocyte dysfunction and cell death [reviewed in Dietrich et al. (2010)]. Rodent models were employed to demonstrate protection from FSGS-like symptoms by TRPC6 deletion (Eckel et al., 2011; Spires et al., 2018; Wang et al., 2019) or inactivation (Kim et al., 2018), as well as to dissect cellular pathways activating TRPC6 channels (Ilatovskaya et al., 2018). Along these lines, podocyte-specific expression of TRPC6 was sufficient to cause glomerular disease in mice (Krall et al., 2010). However, attempts to express FSGS mutant in wild-type as well as in TRPC6-deficient primary podocytes by lentiviral transfer always caused immediate cell death (Kalwa et al., 2015). Therefore, it remains elusive if TRPC6 mutations in podocytes or in any other cell type of the glomerulus are responsible for the development of FSGS in human patients.

Molecular structures of homotetrameric human (Tang et al., 2018) and murine (Azumaya et al., 2018) TRPC6 channels were recently resolved by cryoelectron microscopy at 3.8-Å resolution. Ankyrin repeats at the amino-terminus together with carboxyl-terminus-coiled coil domains are involved in the assembly of the tetramer, which forms a transmembrane layer held by a bell-shaped cytosolic layer. However, 84 amino-terminal amino acids of the human (Tang et al., 2018) as well as 92 amino acids of the murine TRPC6 protein (Azumaya et al., 2018) were not resolved, and it remains unclear if and how the distal amino-terminus interacts with the carboxyl-terminus of the channel. Although the human TRPC6 was complexed with a specific inhibitor (BTDM), which binds between the S5 and S6 domains blocking the channel pore (Tang et al., 2018), activation by the permeable analog of diacylglycerol, 1-oleoyl-1-acetyl-sn-glycerol (OAG), was likewise not analyzed for all TRPC channels. Therefore, we set out to quantify changes in amino- and carboxyl-terminal interactions of the TRPC6 channel after OAG activation by Förster resonance energy transfer (FRET). We added a tetracysteine motif to the amino-terminal end that binds to the small fluorescein arsenical hairpin binder (FlAsH) to quantify interactions more efficiently by minimizing perturbation of protein function (Hoffmann et al., 2005), which often occurs after addition of large tags like cyan or yellow fluorescent proteins. FlAsH-tagged TRPC6 channels were translocated to the plasma membrane and activated like wild-type (WT) channels by OAG. We were able to detect a decrease in FRET ratios after adding OAG to human embryonic kidney (HEK) 293T cells expressing WT and gain- and loss-of-function mutants of TRPC6. In the mutants, however, changes in FRET ratios were significantly reduced, pointing to an important function of amino- to carboxyl-terminal interactions during TRPC6 channel activation by OAG. Our data show for the first time that FlAsH-tagging of ion channels is an important tool for studying conformational changes after channel opening and will significantly advance the analysis of the mechanisms of ion channel activation of TRPC6 mutants expressed in FSGS patients.

\section{Materials and Methods}

Generation of FRET Constructs and Murine TRPC6 FSGS Mutants. FRET constructs of mouse TRPC6 were designed by inserting cDNAs coding for mTRPC6 into the pcDNA3.1 vector containing the cyan fluorescent protein cerulean. New restriction sites for KpnI in the mTRPC6 cDNA coding for the amino-terminal and BamHI in the coding region for the carboxyl-terminus were inserted by polymerase chain reaction using the following primer pairs: 5'-GAC TAG GTA CCA TGA GCC AGA GCC CGA GGT T-3' (sense with KpnI site) and 5'-ATA ATG GAT CCC TCT GCG GCT TTC CTC CAG CT-3' (antisense with BamH I site). The cDNA for mTRPC6 with the inserted restriction sites was released from the vector by digestion with KpnI and BamHI (Thermo Fisher Scientific, Waltham) and inserted with T4 Ligase (Thermo Fisher Scientific) in the pcDNA3.1 vector containing the cDNA coding for the Cerulean protein. The resulting construct was linearized with the same restriction enzymes after ligation. Next, a tetracysteine motif coding for the amino acids C C - P G - C C was inserted into the cDNA region coding for the very amino-terminus of TRPC6 via QuikChange II XL site-directed mutagenesis (Agilent, Santa Clara) using the following primer pairs: 5'-CCC AAG CTT GGT ACC ATG TGT TGC CCG GGC TGC TGT AGC CAG AGC CCG AGG TTC G-3' (sense) and 5'-CGA ACC TCG GGC TCT GGC TAC AGC AGC CCG GGC AAC ACA TGG TAC CAA GCT TGG G-3' (antisense), resulting in the intramolecularly labeled FRET sensor TC-mTRPC6-cerulean. Murine homologs of human FSGS mutants of TRPC6 (G108S, M131T) and the N109A mutation were produced via site-directed mutagenesis as described before using the following primer pairs: $5^{\prime}$-CGC TTT CTA GAT GCA GCT GAA TAT TCC AAC ATC CCA GTG GTG CGG-3' (sense G108S), 5'-CCG CAC CAC TGG GAT GTT GGA ATA TTC AGC TGC ATC TAG AAA GCG-3' (antisense G108S), 5'-CGC TTT CTA GAT GCA GCT GAA TAT GGC AAC ATC CCA GTG GTG CGG-3' (sense N109A), 5' CGC TTT CTA GAT GCA GCT GAA TAT GGC GCC ATC CCA GTG GTG CGG-3' (antisense N109A), 5'-GTT AAC TGT GTG GAT TAC ACG GGC CAG AAT GCC CTA CAG C-3' (sense M131T), 5' -GCT GTA GGG CAT TCT GGC CCG TGT AAT CCA CAC AGT TAA C-3' (antisense M131T). All constructs were sequenced for verification of the correct integration and the absence of additional mutations. 
Cell Culture and Transient Transfections of FRET Constructs. HEK293T cells (293T, ATCC CRL-3216) were maintained in Dulbecco's modified Eagle's medium (DMEM; Lonza, Basel, Switzerland) supplemented with $10 \%$ fetal calf serum (FCS; Thermo Fisher Scientific) and $100 \mathrm{IU} / \mathrm{ml}$ penicillin and $100 \mathrm{mg} / \mathrm{ml}$ streptomycin (Lonza) at $37^{\circ} \mathrm{C}$ and $5 \% \mathrm{CO}_{2}$. Cells were seeded into six-well plates and transfected at a cell confluency of about $80 \%$ with $2.5 \mu \mathrm{g}$ of vector DNA using TransIT-2020 (Mirus Bio, Madison). Assays were performed 48-72 hours after transfection. For FRET experiments, transfected cells were seeded on poly-L-lysine (Sigma-Aldrich, St. Louis)-coated six-channel $\mu$-slides 0.4 (ibidi, Martinsried Germany) 16 hours prior to measurements or onto poly-L-lysine-coated coverslips of $30-\mathrm{mm}$ diameter (neoLab, Heidelberg, Germany). For electrophysiological analysis, cells were seeded onto $33-\mathrm{mm}$ culture dishes (Nunclon; Thermo Fisher Scientific) 3 hours prior to experiments.

Confocal Microscopy of Cells Transfected with Tagged TRPC6 Constructs. Transfected cells were seeded on $18 \times 18-\mathrm{mm}$ coverslips and fixated in $3.7 \%$ paraformaldehyde. Cells were stained for 10 minutes with $2 \mu \mathrm{g} / \mathrm{ml}$ of Hoechst dye (Thermo Fisher Scientific), washed $2 \times$ with phosphate-buffered saline and mounted in Vectashield (Vector Laboratories, Burlingame) on glass slides (Carl Roth, Karlsruhe, Germany). Confocal microscopy was performed using a Zeiss LSM 880 with an alpha Plan-Apochromat $40 \times / 1.3$ oil objective (Zeiss, Oberkochen, Germany). For excitation of cerulean and Hoechst dyes, a 458-nm argon laser was used, and emission was analyzed at $463-580 \mathrm{~nm}$ and at $410-447 \mathrm{~nm}$ for cerulean and Hoechst dye, respectively. All parameters of the microscope were kept identical for measurement of the different FRET constructs. For each analyzed cell a $\mathrm{z}$ stack was obtained, and the image with the largest cell perimeter was used for quantification of fluorescence intensities. An outer and an inner perimeter line was drawn along the outer and the inner plasma membrane, respectively. Cerulean fluorescence was quantified between these lines, which were spaced at a distance of $0.21 \mu \mathrm{m}$. For each cell the sum of fluorescence intensities was calculated and divided by the total length of the perimeter to obtain cerulean fluorescence per nanometer of the plasma membrane.

Analysis of OAG Activation of FRET Constructs by Patch Clamp Recordings. HEK293T cells (293T, ATCC CRL-3216) were maintained in Eagle's minimum essential medium (Sigma-Aldrich, Taufkirchen, Germany), with $100 \mathrm{IU} / \mathrm{ml}$ of penicillin and $100 \mu \mathrm{g} / \mathrm{ml}$ of streptomycin supplemented with $10 \%$ (vol/vol) FCS (Gibco, Thermo Fisher Scientific) and $2 \mathrm{mM}$ glutamine. All cells were held at $37^{\circ} \mathrm{C}$ in a humidified atmosphere with $5 \% \mathrm{CO}_{2}$. Cells were transiently transfected at confluency of about $90 \%$ using GeneJuice (Merck Millipore, Billerica) according to the manufacturer's protocol. Conventional whole-cell recordings were carried out at room temperature 72 hours after transfection of the cells with the mouse TRPC6 FRET constructs TRPC6 WT, TRPC6 M131T, TRPC6 G108S, or TRPC6 N109A. Wholecell recordings of HEK293T cells transfected with the untagged mouse TRPC6 WT or TRPC6 M131T cDNAs in a bicistronic pIRES2-EGFP expression vector were analyzed 48 hours after transfection. A bath solution containing $140 \mathrm{mM} \mathrm{NaCl}, 5 \mathrm{mM} \mathrm{CsCl}, 1 \mathrm{mM} \mathrm{MgCl} \mathrm{MM}_{2}, 2 \mathrm{mM}$ $\mathrm{CaCl}_{2}, 10 \mathrm{mM}$ glucose, and $10 \mathrm{mM}$ HEPES (pH 7.4 with $\mathrm{NaOH}$ ) and resulting in an osmolality of $295-302 \mathrm{mOsm} \mathrm{kg}^{-1}$ was used for patchclamp recordings. The pipette solution contained $120 \mathrm{mM} \mathrm{CsCl}$, $9.4 \mathrm{mM} \mathrm{NaCl}, 0.2 \mathrm{mM} \mathrm{Na}$-GTP, $1 \mathrm{mM} \mathrm{MgCl}_{2}, 3.949 \mathrm{mM} \mathrm{CaCl}_{2}$, $10 \mathrm{mM}$ BAPTA (100 nM free $\mathrm{Ca}^{2+}$ ), and $10 \mathrm{mM}$ HEPES (pH 7.2 with $\mathrm{CsOH}$ ), resulting in an osmolality of $296 \mathrm{mOsm} \mathrm{kg}{ }^{-1}$. Patch pipettes made of borosilicate glass (Science Products, Hofheim, Germany) had resistances of 2.2-3.5 $\mathrm{M} \Omega$ for whole-cell measurements. Data were collected with an EPC10 patch clamp amplifier (HEKA, Lambrecht, Germany) using the Patchmaster software. Current density-voltage relations were obtained from voltage ramps from -100 to $+60 \mathrm{mV}$ with a slope of $0.4 \mathrm{~V} \mathrm{~s}^{-1}$ applied at a frequency of $1 \mathrm{~Hz}$. Data were acquired at a frequency of $5 \mathrm{kHz}$ after filtering at $1.67 \mathrm{kHz}$. The current density-voltage curves and the current density amplitudes at $\pm 60 \mathrm{mV}$ were extracted at minimal or maximal currents, respectively.
Determination of FRET Efficiency. As a measurement of the FRET efficiencies of the different FRET constructs, we determined the recovery of the donor fluorescence (cerulean) after removal of the acceptor fluorophore FlAsH by British anti-Lewisite (BAL) (2,3-dimercapto-1-propanol; Sigma-Aldrich). Transfected cells were seeded on 30-mm-diameter coverslips and labeled with FlAsH. Coverslips were placed in an Attofluor holder with $1 \mathrm{ml}$ Hanks' balanced salt solution (HBSS), and emission wavelengths of cerulean and FlAsH were measured as described for FRET experiments (see below). After steady-state levels for both fluorophores was reached, BAL was added to reach a final concentration of $2 \mathrm{mM}$. FRET efficiency was determined as $\mathrm{E}=1-\left(\operatorname{tau}_{\mathrm{DA}} / \mathrm{tau}_{\mathrm{D}}\right)$, in which tau $\mathrm{DA}_{\mathrm{DA}}$ is the fluorescence of the donor in the presence of the acceptor and tau $\mathrm{D}_{\mathrm{D}}$ is the fluorescence of the donor in absence of the acceptor.

FlAsH-Labeling and FRET Measurements. Transfected HEK293T cells grown on $\mu$-slides were washed twice with $100 \mu \mathrm{l}$ of phenol red-free $\mathrm{HBSS}$ (Lonza) and incubated at $37^{\circ} \mathrm{C}$ and $5 \% \mathrm{CO}_{2}$ for 1 hour with $100 \mu \mathrm{l}$ of $1 \mu \mathrm{M}$ FlAsH-EDT ${ }_{2}$-labeling reagent in HBSS (Thermo Fisher Scientific). After labeling, cells were washed once with $100 \mu \mathrm{l} \mathrm{HBSS}$. To reduce the background signal, cells were incubated at $37^{\circ} \mathrm{C}$ and $5 \% \mathrm{CO}_{2}$ for 10 minutes with $100 \mu \mathrm{l}$ of $0.25 \mathrm{mM}$ $\mathrm{EDT}_{2}$ in HBSS (Sigma-Aldrich). Finally, cells were washed twice with HBSS and HEPES-buffered DMEM without phenol red was added. For cells grown on $30-\mathrm{mm}$ diameter coverslips, $1 \mathrm{ml}$ of working volume was used. FRET experiments were performed on an Olympus IX70 inverted microscope equipped with an UPlanSAPO $100 \times / 1.40$ oil objective (Olympus, Hamburg, Germany) and connected to an dual-emission photometry system (TILL Photonics, Martinsried, Germany). Cells were excited at a wavelength of $430 \mathrm{~nm}$ produced by a Polychrome V (TILL Photonics) and emission spectra for cerulean at $480 \pm 20$ as well as for FlAsH at $535 \pm 15 \mathrm{~nm}$ were measured by a dual-emission photometry system using a beam splitter dichroic long pass $505 \mathrm{~nm}$. Emissions were quantified as voltage of the transimpedance amplifier of the photodiodes with a frequency of $10 \mathrm{kHz}$ and were collected by an EPC10 amplifier (HEKA) with the PATCHMASTER software (HEKA). Excitation time was $4.6 \mathrm{~ms}$ and sampling rate was $10 \mathrm{~Hz}$. Fluorescence traces were not corrected for photo-bleaching since stimuli were only applied when constant fluorescence values were attained. Cells were constantly perfused with phenol red-free HBSS (Lonza) during FRET measurements. After steady-state levels of the fluorophores were reached, the dimethyl sulfoxide (DMSO) control was applied followed by application of $100 \mu \mathrm{M}$ OAG (Merck, Darmstadt, Germany) or $10 \mu \mathrm{M}$ GSK-1702934A (GSK-170) (Focus Biomolecules, Pennsylvania) in DMSO. FRET was determined as the emission ratio of FlAsH to cerulean upon TRPC6 activation by OAG or GSK-170.

The three criteria applied to obtain valid FRET measurements were as follows: 1) Only FRET measurements that showed a ratio of FlAsH to cerulean fluorescence of $1: 2$ to $1: 4$ were included in the analysis. This criterion helped to exclude cells with unspecific FlAsH labeling. 2) Only FRET measurements that developed decreasing FlAsH and increasing cerulean fluorescence were further evaluated. This criterion enhanced the reliability and validity of the obtained data. 3) Only FRET measurements that showed no changes in FRET signals during the application of the solvent were included in the presented data set.

Statistical Analysis. Data were analyzed using Origin 7.5 software (OriginLab, Northampton) and GraphPad Prism 7 software (GraphPad, San Diego). We chose to use one-way analysis of variance (ANOVA) for multiple comparisons before generating the data. We first tested the data for Gaussian distribution using the Shapiro-Wilk test, and if assumed we applied one-way ANOVA. If variability between groups was greater than within groups a Tukey-Kramer post-test was applied. If Gaussian distribution was not assumed, nonparametric tests (Wilcoxon matched-pairs signed-rank test or Mann-Whitney $U$ test) were used. Data are 
A

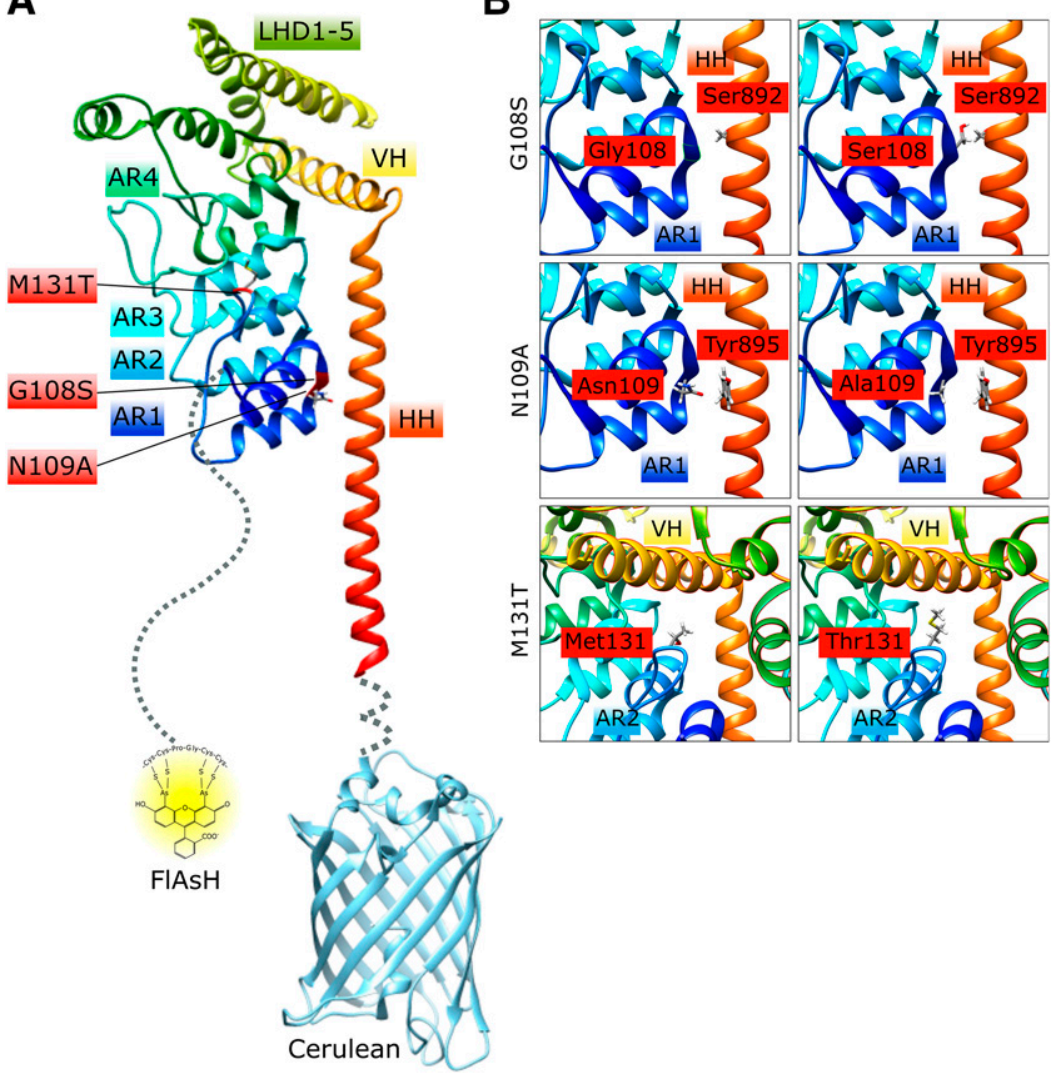

Fig. 1. Structures of TRPC6 amino and carboxyl termini, tagging strategy (A), and localization of FSGS mutants M131T, G108S, as well as the newly established mutant N109A (A and B). Protein structures are labeled according to Azumaya et al. (2018) and were visualized with the UCSF Chimera software by usage of the Protein Data Bank ID: 6CV9. AR, ankyrin repeat; HH, horizontal helix; LHD, linker helical domain; VH, vertical helix. presented as median \pm interquartile ranges or means \pm S.D. if Gaussian distribution was assumed.

\section{Results}

Tagging Strategies for TRPC6 WT and Mutant Proteins to Analyze Carboxyl- and Amino-Terminal Interactions by FRET. We constructed tagged murine TRPC6 wild-type and mutant proteins by adding a tetracysteine motif coding for the amino acids $2 \times \mathrm{C}$ (for cysteine), $\mathrm{P}$ (for proline), $\mathrm{G}$ (for glycine), and again $2 \times \mathrm{C}$ to the coding region of the end of the amino-terminus as well as the cDNA for the cerulean protein to the end of the carboxyl-terminus (Fig. 1A). This strategy was already successfully tested for $G$ protein-coupled receptors (GPCR) and was very helpful in the monitoring of conformational changes during agonist-induced GPCR activation in living cells (Hoffmann et al., 2005). The first 92 amino acids of the amino-terminus and the last nine amino acids of the carboxyl-terminus were not resolved in the recent analysis of the murine TRPC6 structure (Azumaya et al., 2018) and are therefore dashed in Fig. 1A. We focused our analysis on two already described mutants in the aminoterminus of TRPC6. TRPC6 M131T carries the homologous human M132T gain of function mutation, which showed the highest mean inward current amplitude of all identified mutations in human FSGS patients (Heeringa et al., 2009) and lies on a position in the amino-terminus clearly directed away from the carboxyl-terminus (Azumaya et al., 2018) (Fig. 1). The TRPC6 G108S (human G109S) mutant lies in the amino-terminal region in close proximity to the carboxylterminus (Azumaya et al., 2018) (Fig. 1) and was predicted to be a loss of function FSGS mutation (Santín et al., 2009). For FRET measurements and quantification of channel activity, we also tested a new third mutation, N109A, as N109 forms a strong contact with the amino acid Y895 of the carboxylterminus (Azumaya et al., 2018) (Fig. 1) .

Expression of Tagged TRPC6 Constructs at the Plasma Membrane. To verify translocation of TRPC6 FRET constructs to the plasma membrane, we analyzed by confocal laser microscopy the fluorescence emitted by the cerulean tag at the carboxyl-terminus of the tagged TRPC6 constructs, which revealed their expression in HEK293T cells after transient transfection. The cellular distribution of all three tagged TRPC6 mutants was indistinguishable from the tagged wild-type channel, and the overlay of fluorescence with differential-interference contrast images demonstrated that all TRPC6 proteins were correctly targeted to the plasma membrane (Fig. 2A). Moreover, quantification of tagged TRPC6 constructs revealed similar levels in the plasma membrane of the transfected cells (Fig. 2B).

Patch-Clamp Recordings of Basal and OAG-Induced Current Densities of WT- and Mutant-Tagged TRPC6 Channels. To test channel activities of the tagged TRPC6 WT and mutant FRET constructs, we performed electrophysiological whole-cell measurements of HEK293T cells after transient transfection (Fig. 3; Supplemental Table 1). All constructs were functionally expressed and showed OAGinduced current increases with current density-voltage relationships characteristic of TRPC6 currents (Dietrich et al., 2003). Basal and OAG-induced current densities at $\pm 60 \mathrm{mV}$ of the TRPC6 M131T construct were significantly increased compared with the TRPC6 WT construct, confirming that this 
A

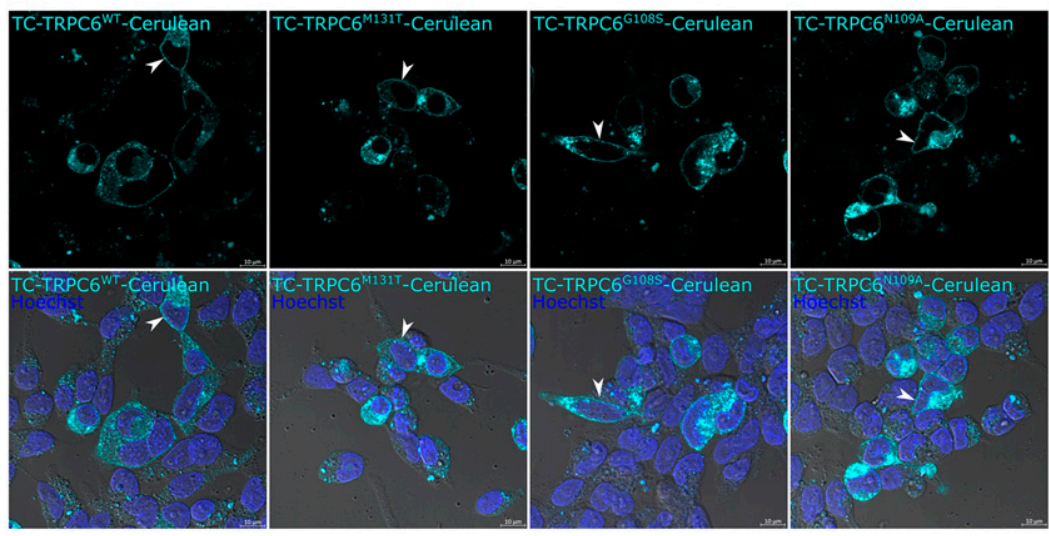

Fig. 2. Subcellular localization (A) and quantification of tagged TRPC6 WT channel and mutants at the plasma membrane (B). Cerulean (cyan)-tagged TRPC6 WT and TRPC6 mutants were transiently transfected into HEK293T cells and analyzed by confocal laser scanning microscopy. Cell nuclei were stained with Hoechst dye (blue). Images showing cerulean fluorescence (upper row) as well as overlays of these pictures with corresponding digital interference contrast (DIC) images (lower row) are depicted. (B) Quantification of tagged WT channels and mutants at the plasma membrane. Shown are medians and interquartile ranges. Non-Gaussian distributed data (ShapiroWilk test) were further analyzed by a Kruskal-Wallis test and no significant differences were identified.

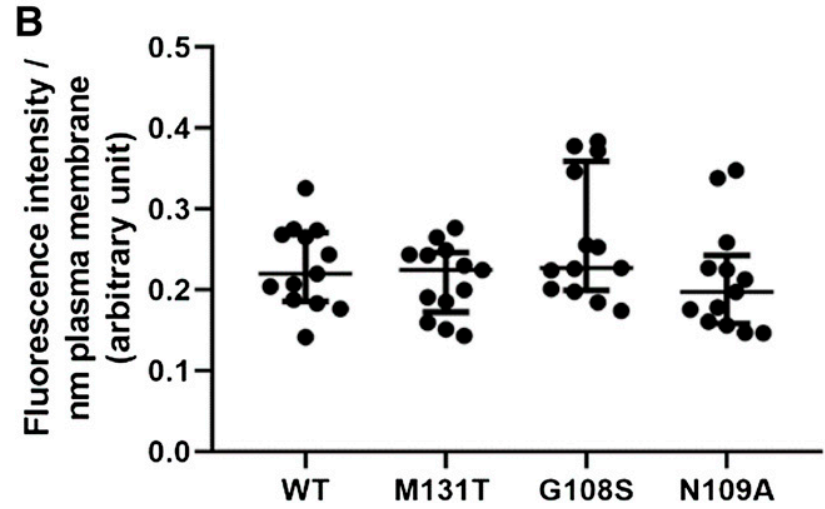

mutation was activated and resulted in a GOF phenotype (Heeringa et al., 2009). To test if protein tags interfere with channel properties, we heterologously expressed untagged WT and M131T mutant TRPC6 channels in HEK293T cells. Basal and OAG-induced current densities at $\pm 60 \mathrm{mV}$ of the TRPC6 M131T GOF mutant were significantly increased compared with the TRPC6 WT channel just like the tagged WT and M131T constructs designed for the FRET analysis (Supplemental Fig. 1; Supplemental Table 2). These findings suggest that our amino- and carboxyl-terminal tags do not substantially influence the gating properties of the analyzed TRPC6 constructs. In contrast, the TRPC6 G108S mutant showed significantly decreased OAG-induced current densities at $\pm 60 \mathrm{mV}$, pointing to an LOF phenotype, which was initially predicted after its identification (Santín et al., 2009). Recently, the G108S mutant was characterized as a GOF mutation in calcium imaging experiments (Riehle et al., 2016). However, our electrophysiological recordings confirmed the initially predicted LOF phenotype. Moreover, the TRPC6 N109A mutation in the amino-terminus in close proximity to the carboxyl-terminus (Tang et al., 2018) showed significantly decreased basal current densities at $+60 \mathrm{mV}$ and significantly decreased OAG-induced current densities at $\pm 60 \mathrm{mV}$. Thus, we were able to identify the N109A mutant as a novel LOF mutation.

Analyzing FRET Efficiencies of WT and MutantTagged TRPC6 Proteins. To investigate the overall quality and comparability of FRET of the different tagged TRPC6 constructs, we analyzed FRET decreases after application of the arsenic molecule by the chelator molecule $\mathrm{BAL}$ (dimercaprol) which led to release of FlAsH from the tetracysteine binding site in transiently transfected HEK293T cells. All tagged TRPC6 constructs showed comparable FRET efficiencies (Fig. 4) as an important requirement for FRET studies after application of OAG.

Quantifying OAG-Induced Interactions of Amino and Carboxyl Termini by FRET in TRPC6 WT and Mutant Channels. We performed FRET measurements next with HEK293T cells transiently transfected with tagged TRPC6 WT or mutant constructs. After application of OAG we observed a decrease in FRET ratios in both TRPC6 WT and mutant constructs, whereas no changes occurred after adding the solvent (0.1\% DMSO) alone (Fig. 5, A-D). Decreases in FRET ratios were, however, significantly reduced in TRPC6 GOF and LOF mutants compared with TRPC6 WT channels (Fig. 5E). Surprisingly, no differences in FRET ratios were detected in all mutants regardless if they were LOF and located in close proximity (G108S, N109A) or GOF and directed away (M131T) from the C-terminus. Similar results were obtained with the nonlipid activator of TRPC6 channels GSK-170 (Fig. 6). Notably, the kinetics of the OAG- and GSK-induced FRET signals was 3- to 4-fold slower than the kinetics of the OAG-induced current increases (Supplemental Fig. 2) and increases in FRET signals were not reversible after washout of the channel activators. However, the latency periods between application of the activator and the development of changes in FRET or increases in current densities were similar. These findings indicate that we were very probably monitoring TRPC6 channel activation. An explanation for the lack of reversibility of FRET signals after removal of TRPC6 activators (OAG and GSK) is still elusive and needs to be analyzed in detail in further studies. 


\section{A TRPC6 WT}

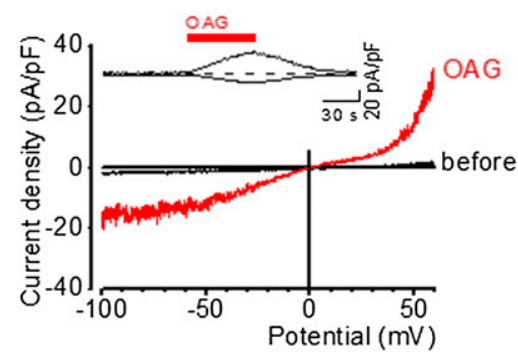

\section{TRPC6 G108S}

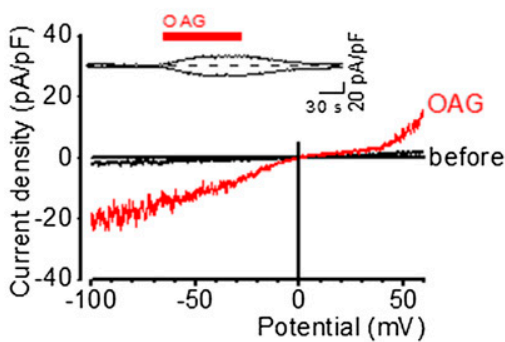

E

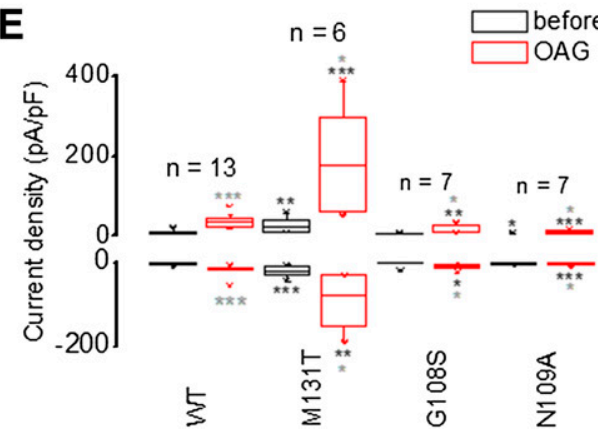

\section{B TRPC6 M131T}

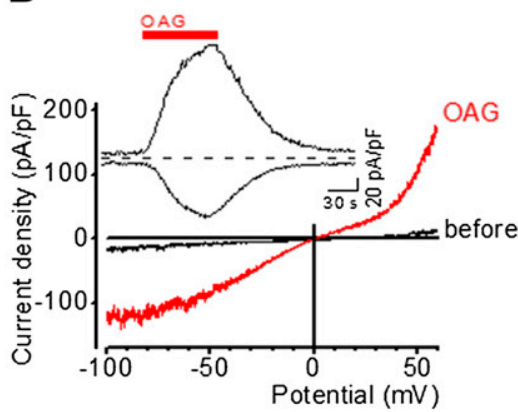

D TRPC6 N109A

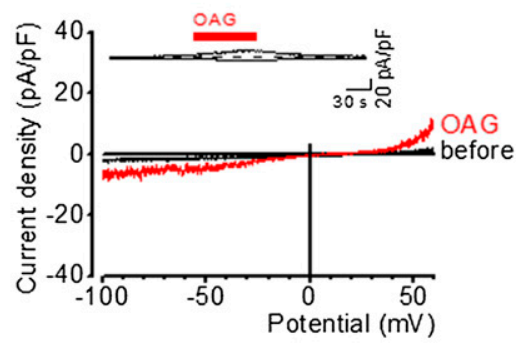

Fig. 3. Patch-clamp recordings of basal and OAG-induced current densities of WT- and mutant-tagged TRPC6 channels. Electrophysiological whole-cell measurements of HEK293T cells expressing indicated FRET constructs (A-E). Representative current density-voltage curves of the wild-type TRPC6 FRET construct (TRPC6 WT) (A) and of the mutant TRPC6 FRET constructs TRPC6 M131T (B), TRPC6 G108S (C), and TRPC6 N109A (D) before (black traces) and during application of $100 \mu \mathrm{M}$ OAG (red traces). Insets show current density-time courses at holding potentials of $\pm 60 \mathrm{mV}$. Application of OAG is indicated. Summary of current densities at $\pm 60 \mathrm{mV}$ before (basal) and during application of OAG displayed as boxplots with interquartile ranges (E). Numbers indicate analyzed cells from at least three independent experiments. As data showed no Gaussian distribution (Shapiro-Wilk test), significance was tested and compared with basal current densities using a Wilcoxon matched-pairs signed-rank test; $* P<0.05$; $* * * P<0.001$ ), gray asterisks) as well as to TRPC6 WT (Mann-Whitney $U$ test, ${ }^{*} P<0.05$; $\left.{ }^{* *} P<0.01^{* * *}, P<0.001\right)$, black asterisks).

\section{Discussion}

Elucidation of protein structures of ion channels is of utmost importance to the study of conformational changes and prediction of regions essential for channel opening. While crystallography of whole TRP proteins turned out to be successful only for TRPV6 (Saotome et al., 2016), recent advances in cryoelectron microscopy have provided the scientific community with highly resolved protein structures of many different TRP ion channels [e.g., TRPV1 (Liao et al., 2013), TRPA1 (Paulsen et al., 2015), TRPC3 and TRPC6 (Tang et al., 2018), murine TRPC6 (Azumaya et al., 2018), TRPM2 (Wang et al., 2018), and TRPM7 (Duan et al., 2018)]. The recently published structures of human and murine TRPC6, however, did not resolve the whole aminoterminus of the protein and to identify conformational changes during activation by its membrane-permeable activator OAG or the nonlipid activator GSK-170. Moreover, it remained elusive how GOF or LOF mutations in the TRPC6 channel identified in patients with FSGS change the overall protein structure and channel activity. All of these FSGS mutations in the TRPC6 protein are located in the amino- or the carboxyl-terminal cytosolic tail of the channel, raising the intriguing possibility that they may change channel activity by altered interactions of both termini. Moreover, most of the amino-terminal mutations are located in predicted ankyrin-repeats of the TRPC6 channel. Ankyrin repeats have important ligand binding properties (Gaudet, 2008), mediate protein-protein interactions ( $\mathrm{Li}$ et al., 2006), and have been implicated in mechanosensation (Zhang et al., 2015). Therefore, we set out to quantify amino- and carboxyl-terminal interactions during OAG activation of WT and mutant TRPC6 channels by FRET in an exploratory study. We chose a very small fluorescent marker at the amino-terminus called FlAsH to avoid perturbations of conformational changes and an efficient fluorescent partner protein cerulean, which had already been used successfully for analyzing conformational changes in GPCRs during activation by their agonists (Hoffmann et al., 2005) (Fig. 1). Tagged channels were efficiently translocated to the plasma membrane (Fig. 2) and revealed predicted channel activities quantified by patch clamp recording (Fig. 3). Molecules at both termini interacted closely and exhibited similar FRET efficiency (Fig. 4). After application of OAG or GSK-170, FRET ratios decreased for TRPC6 WT and mutant TRPC6 channels, but the FRET signal decreases were significantly lower in the analyzed mutant channels (Figs. 5 and 6). For the first time, our results show conformational changes of 

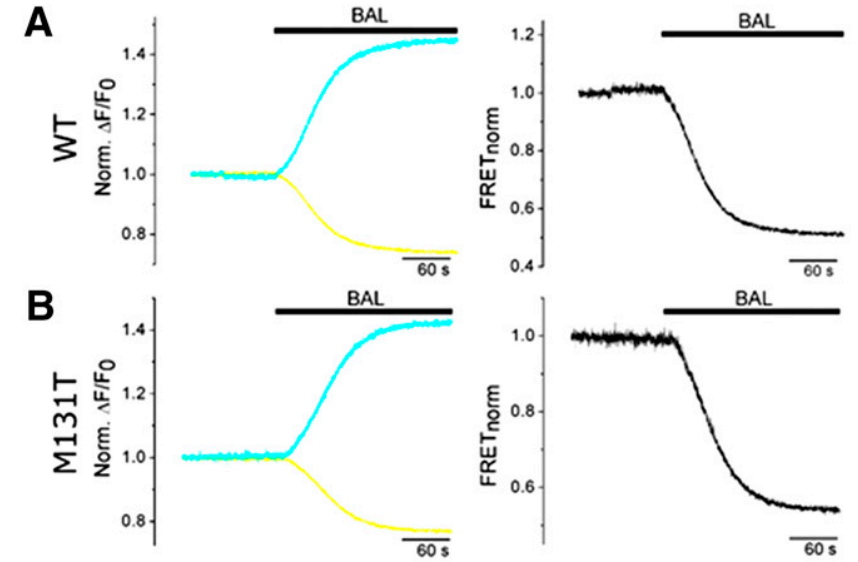

C
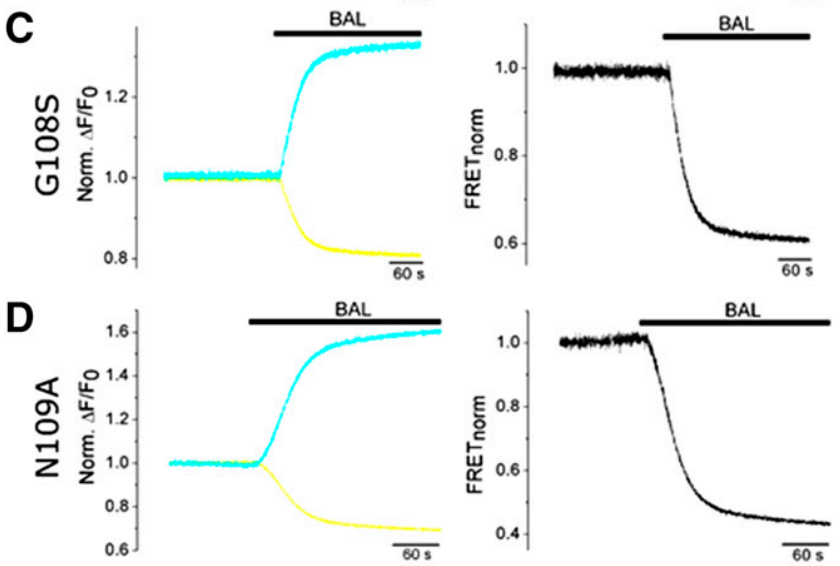

E

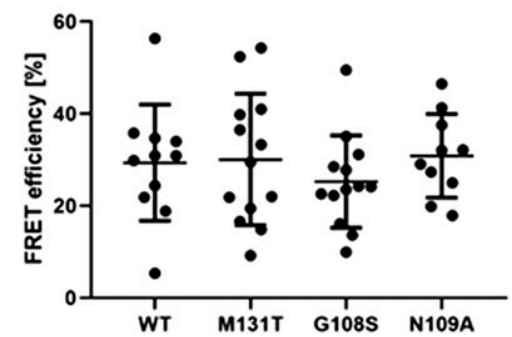

Fig. 4. Quantification of FRET efficiencies of tagged TRPC6 WT channels and mutants. FRET efficiencies were analyzed in HEK293T cells expressing TRPC6 WT or mutants after application of British anti-Lewisite (BAL). Representative normalized ratios of changes in fluorescence emission signals from cerulean (cyan) and tC-FlAsH (yellow) [norm. $\Delta \mathrm{F} / \mathrm{F}_{0}$, (A-D), left] and normalized FRET ratios [FRET norm., (A-D), right] are depicted. (E) Summary of FRET efficiencies quantified by removal of arsenic from FlAsH by BAL. Gaussian distributed data (ShapiroWilk test) were further analyzed by one-way ANOVA and no significant differences were identified.

amino- to caboxyl-terminal interactions in the homotetrameric TRPC6 channel occurring during channel activation by OAG or GSK-170, as detected by the FRET technology using FlAsH and cerulean as interaction partners.

Similar results were reported for TRPV4 channels expressing cyan fluorescent and yellow fluorescent proteins at their amino and carboxyl termini, respectively (Hellwig et al., 2005; Strotmann et al., 2010). Application of a hypotonic medium or of receptor agonists like carbachol resulted in transient decreases in FRET signals (Strotmann et al., 2010). Moreover, mutations in the amino-terminal regions of TRPV4 reduced interactions with the carboxylterminus and increased $\mathrm{Ca}^{2+}$ /calmodulin (CaM)-dependent
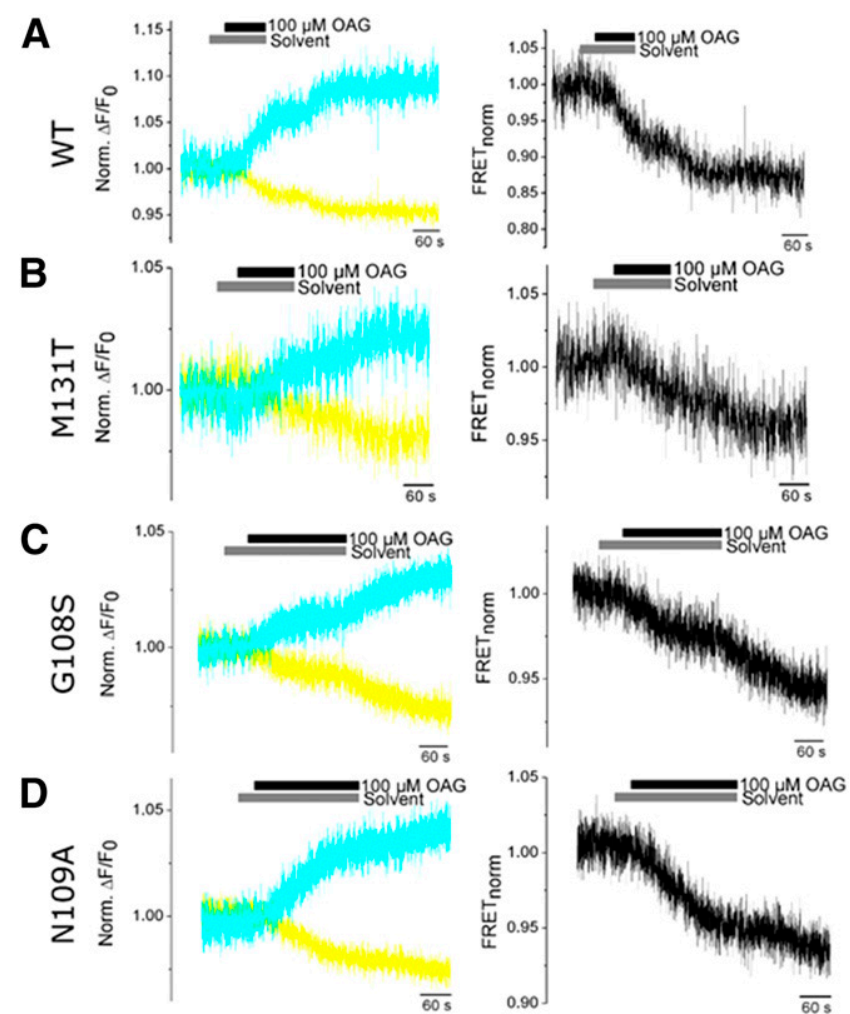

E

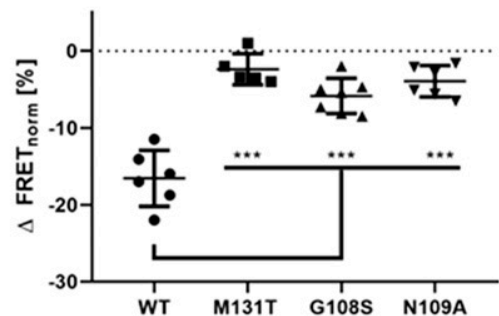

potentiation of the channel, whereas amino acid changes in the calmodulin binding domain preventing CaM binding reduced channel activity (Strotmann et al., 2010). Although TRPC6 channel activity was predicted to be inhibited and not potentiated by $\mathrm{Ca}^{2+}$ /calmodulin at a different binding site than that reported for TRPC3 (Zhang et al., 2001), amino- to carboxyl-terminal interactions during activation seem to be conserved in both channels.

In the recently resolved protein structure of TRPM2 by cryoelectron microscopy, the carboxyl-terminal NUDT9 homology domain interacts with both inter- and intramolecular amino termini (Wang et al., 2018). Moreover, the authors proposed a model wherein the amino-terminal TRPM homology region is removed from the neighborhood of the carboxyl-terminus by rotation during binding of the channel activator ADP-ribose and opening of the channel (Wang et al., 2018). Although TRPC6 does not express an NUDT9 

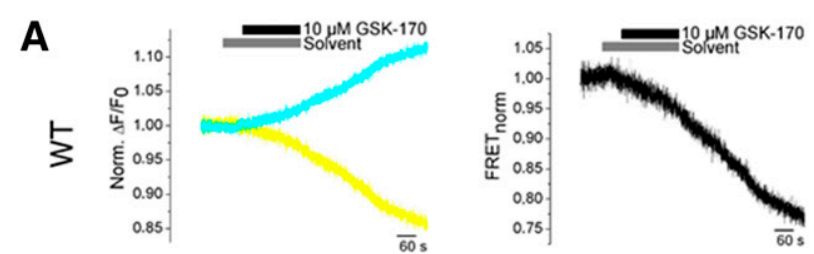

\section{B}
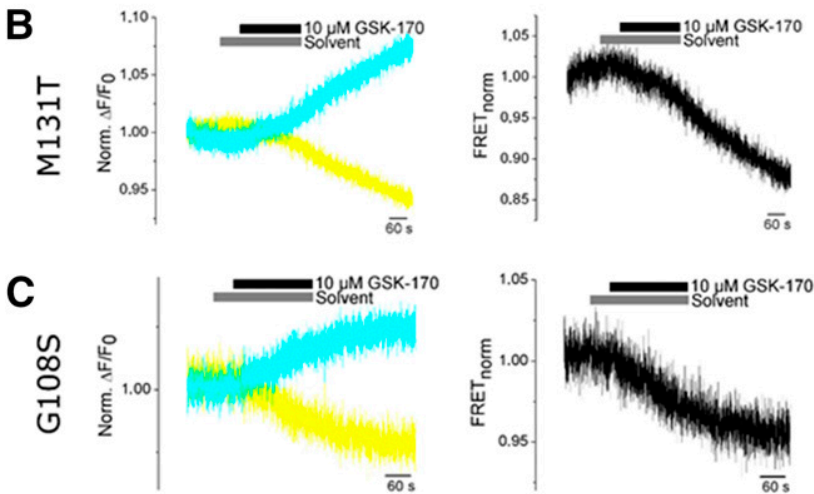

\section{D
बू
ํ.}
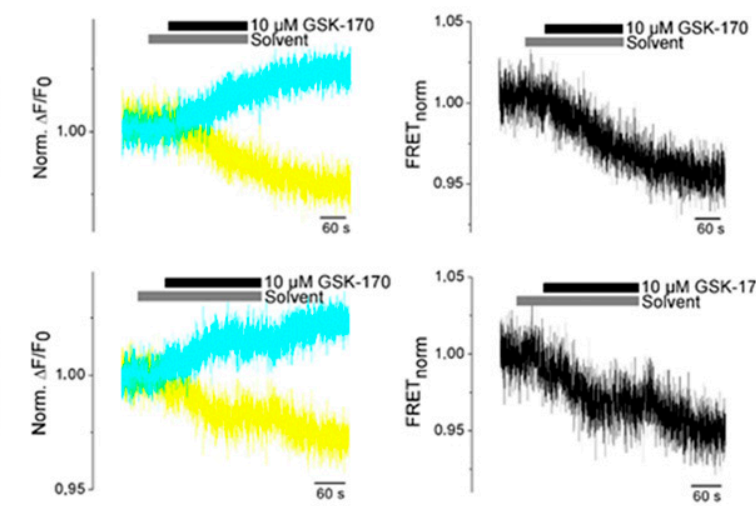

E

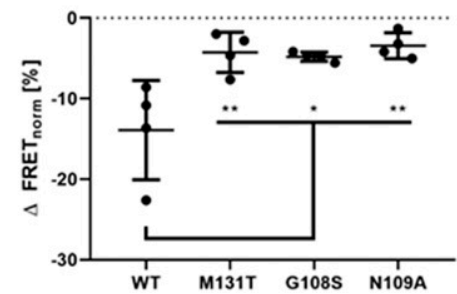

Fig. 6. Quantifying GSK-170-induced changes in FRET of TRPC6 WT and mutant channels. FRET was analyzed in HEK293T cells transfected with TRPC6 WT or TRPC6 mutants during activation by GSK-170. Representative normalized ratios of changes in fluorescence emission signals from cerulean (cyan) and FlAsH (yellow) [norm. $\Delta \mathrm{F} / \mathrm{F}_{0},(\mathrm{~A}-\mathrm{D})$, left] and normalized FRET ratios [FRET norm., (A-D), right] are depicted. Summary of decreases in FRET ratios induced by GSK-170 (E). Data are shown as mean \pm S.D. Gaussian distributed data (Shapiro-Wilk test) were further analyzed by one-way ANOVA and a Tukey-Kramer post-test was applied. ${ }^{*} P<0.05,{ }^{*} P P<0.01$.

homology domain and is not activated by ADP-ribose, our data are suggestive of a similar conformational change in the amino-terminus of this channel. In TRPC6 WT channels the amino-terminus may be moved away from the carboxyl-terminus by a conformational change during channel activation, and channel opening results in a decrease in the FRET signal. GOF mutations (M131T) may induce a preactivated channel with higher basal activity (see Fig. 3) in which the amino-terminus is already partially removed from the carboxyl-terminus. Therefore, OAG activation results in a significantly smaller decrease in the FRET signal but higher channel activity. LOF mutations (G108S and the newly established N109A) may have lost the ability for a full conformational change after OAG binding. In these LOF TRPC6 channels OAG binding might induce only a small conformational change, moving the amino-terminus partially away from carboxyl-terminus and resulting in a reduced FRET decrease as for the GOF mutations, but with much lower channel activity. Thus, this model would explain why LOF and GOF mutations, which change the overall structure of the amino-terminus regardless of whether their location is in close proximity to or directed away from the C-terminus, induce likewise reduced decreases in FRET despite their different channel activities.

Altogether, dynamic FRET with FlAsH-tagged ion channels is a promising novel tool for quantifying conformational changes in the protein structure of TRPC6 and other ion channels during activation.

\section{Authorship Contributions}

Participated in research design: Fiedler, Storch, Mederos y Schnitzler, Dietrich.

Conducted experiments: Fiedler, Storch.

Contributed new reagents or analytic tools: Erdogmus.

Performed data analysis: Fiedler, Storch, Mederos y Schnitzler, Dietrich.

Wrote or contributed to the writing of the manuscript: Fiedler, Storch, Mederos y Schnitzler, Gudermann, Dietrich.

\section{References}

Azumaya CM, Sierra-Valdez F, Cordero-Morales JF, and Nakagawa T (2018) Cryo-EM structure of the cytoplasmic domain of murine transient receptor potential cation channel subfamily C member 6 (TRPC6). J Biol Chem 293:10381-10391.

Boulay G, Zhu X, Peyton M, Jiang M, Hurst R, Stefani E, and Birnbaumer L (1997) Cloning and expression of a novel mammalian homolog of Drosophila transient receptor potential (Trp) involved in calcium entry secondary to activation of receptors coupled by the Gq class of G protein. J Biol Chem 272:29672-29680.

Dietrich A, Chubanov V, and Gudermann T (2010) Renal TRPathies. J Am Soc Nephrol 21:736-744.

Dietrich A, Fahlbusch M, and Gudermann T (2014) Classical transient receptor potential 1 (TRPC1): channel or channel regulator? Cells 3:939-962.

Dietrich A and Gudermann T (2014) TRPC6: physiological function and pathophysiological relevance. Handb Exp Pharmacol 222:157-188.

Dietrich A, Kalwa H, Rost BR, and Gudermann T (2005a) The diacylgylcerolsensitive TRPC3/6/7 subfamily of cation channels: functional characterization and physiological relevance. Pflugers Arch 451:72-80.

Dietrich A, Mederos y Schnitzler M, Emmel J, Kalwa H, Hofmann T, and Gudermann $\mathrm{T}$ (2003) N-linked protein glycosylation is a major determinant for basal TRPC3 and TRPC6 channel activity. J Biol Chem 278:47842-47852.

Dietrich A, Mederos y Schnitzler M, Kalwa H, Storch U, and Gudermann T (2005b) Functional characterization and physiological relevance of the TRPC3/6/7 subfamily of cation channels. Naunyn Schmiedebergs Arch Pharmacol 371:257-265.

Duan J, Li Z, Li J, Hulse RE, Santa-Cruz A, Valinsky WC, Abiria SA, Krapivinsky G, Zhang J, and Clapham DE (2018) Structure of the mammalian TRPM7, a magnesium channel required during embryonic development. Proc Natl Acad Sci USA 115:E8201-E8210.

Eckel J, Lavin PJ, Finch EA, Mukerji N, Burch J, Gbadegesin R, Wu G, Bowling B, Byrd A, Hall G, et al. (2011) TRPC6 enhances angiotensin II-induced albuminuria. J Am Soc Nephrol 22:526-535.

Gaudet R (2008) A primer on ankyrin repeat function in TRP channels and beyond. Mol Biosyst 4:372-379.

Heeringa SF, Möller CC, Du J, Yue L, Hinkes B, Chernin G, Vlangos CN, Hoyer PF, Reiser J, and Hildebrandt F (2009) A novel TRPC6 mutation that causes childhood FSGS. PLoS One 4:e7771.

Hellwig N, Albrecht N, Harteneck C, Schultz G, and Schaefer M (2005) Homo- and heteromeric assembly of TRPV channel subunits. J Cell Sci 118:917-928.

Hoffmann C, Gaietta G, Bünemann M, Adams SR, Oberdorff-Maass S, Behr B, Vilardaga JP, Tsien RY, Ellisman MH, and Lohse MJ (2005) A FlAsH-based FRET approach to determine $\mathrm{G}$ protein-coupled receptor activation in living cells. Nat Methods 2:171-176.

Hofmann T, Obukhov AG, Schaefer M, Harteneck C, Gudermann T, and Schultz G (1999) Direct activation of human TRPC6 and TRPC3 channels by diacylglycerol. Nature 397:259-263.

Ilatovskaya DV, Blass G, Palygin O, Levchenko V, Pavlov TS, Grzybowski MN, Winsor K, Shuyskiy LS, Geurts AM, Cowley AW Jr, et al. (2018) A NOX4/TRPC6 pathway in podocyte calcium regulation and renal damage in diabetic kidney disease. J Am Soc Nephrol 29:1917-1927.

Kalwa H, Storch U, Demleitner J, Fiedler S, Mayer T, Kannler M, Fahlbusch M, Barth H, Smrcka A, Hildebrandt F, et al. (2015) Phospholipase C epsilon (PLC $\varepsilon$ ) induced TRPC6 activation: a common but redundant mechanism in primary podocytes. J Cell Physiol 230:1389-1399.

Kim EY, Yazdizadeh Shotorbani P, and Dryer SE (2018) Trpc6 inactivation confers protection in a model of severe nephrosis in rats. J Mol Med (Berl) 96:631-644.

Krall P, Canales CP, Kairath P, Carmona-Mora P, Molina J, Carpio JD, Ruiz P, Mezzano SA, Li J, Wei C, et al. (2010) Podocyte-specific overexpression of wild type or mutant trpc6 in mice is sufficient to cause glomerular disease. PLoS One 5: e12859.

Li J, Mahajan A, and Tsai MD (2006) Ankyrin repeat: a unique motif mediating protein-protein interactions. Biochemistry 45:15168-15178. 
Liao M, Cao E, Julius D, and Cheng Y (2013) Structure of the TRPV1 ion channel determined by electron cryo-microscopy. Nature 504:107-112.

Mottl AK, Lu M, Fine CA, and Weck KE (2013) A novel TRPC6 mutation in a family with podocytopathy and clinical variability. BMC Nephrol 14:104.

Paulsen CE, Armache JP, Gao Y, Cheng Y, and Julius D (2015) Structure of the TRPA1 ion channel suggests regulatory mechanisms [published correction appears in Nature (2015) 525:552]. Nature 520:511-517.

Reiser J, Polu KR, Möller CC, Kenlan P, Altintas MM, Wei C, Faul C, Herbert S, Villegas I, Avila-Casado C, et al. (2005) TRPC6 is a glomerular slit diaphragm-associated channel required for normal renal function. Nat Genet 37:739-744.

Riehle M, Büscher AK, Gohlke BO, Kaßmann M, Kolatsi-Joannou M, Bräsen JH, Nagel M, Becker JU, Winyard P, Hoyer PF, et al. (2016) TRPC6 G757D loss-of-function mutation associates with FSGS. J Am Soc Nephrol 27: 2771-2783.

Santín S, Ars E, Rossetti S, Salido E, Silva I, García-Maset R, Giménez I, Ruíz P, Mendizábal S, Luciano Nieto J, et al.; FSGS Study Group (2009) TRPC6 mutational analysis in a large cohort of patients with focal segmental glomerulosclerosis. Nephrol Dial Transplant 24:3089-3096.

Saotome K, Singh AK, Yelshanskaya MV, and Sobolevsky AI (2016) Crystal structure of the epithelial calcium channel TRPV6. Nature 534:506-511.

Spires D, Ilatovskaya DV, Levchenko V, North PE, Geurts AM, Palygin O, and Staruschenko A (2018) Protective role of Trpc6 knockout in the progression of diabetic kidney disease. Am J Physiol Renal Physiol 315: F1091-F1097.

Storch U, Forst AL, Pardatscher F, Erdogmus S, Philipp M, Gregoritza M, Mederos Y Schnitzler M, and Gudermann T (2017) Dynamic NHERF interaction with TRPC4/5 proteins is required for channel gating by diacylglycerol. Proc Natl Acad Sci USA 114:E37-E46.

Strotmann R, Semtner M, Kepura F, Plant TD, and Schöneberg T (2010) Interdomain interactions control $\mathrm{Ca} 2+$-dependent potentiation in the cation channel TRPV4. PLoS One 5:e10580.
Tang Q, Guo W, Zheng L, Wu JX, Liu M, Zhou X, Zhang X, and Chen L (2018) Structure of the receptor-activated human TRPC6 and TRPC3 ion channels. Cell Res 28:746-755.

Wang L, Chang JH, Buckley AF, and Spurney RF (2019) Knockout of TRPC6 promotes insulin resistance and exacerbates glomerular injury in Akita mice. Kidney Int 95:321-332.

Wang L, Fu TM, Zhou Y, Xia S, Greka A, and Wu H (2018) Structures and gating mechanism of human TRPM2. Science 362. DOI: 10.1126/science.aav4809.

Weissmann N, Dietrich A, Fuchs B, Kalwa H, Ay M, Dumitrascu R, Olschewski A Storch U, Mederos y Schnitzler M, Ghofrani HA, et al. (2006) Classical transient receptor potential channel 6 (TRPC6) is essential for hypoxic pulmonary vasoconstriction and alveolar gas exchange. Proc Natl Acad Sci USA 103:19093-19098.

Weissmann N, Sydykov A, Kalwa H, Storch U, Fuchs B, Mederos y Schnitzler M, Brandes RP, Grimminger F, Meissner M, Freichel M, et al. (2012) Activation of TRPC6 channels is essential for lung ischaemia-reperfusion induced oedema in mice. Nat Commun 3:649.

Winn MP, Conlon PJ, Lynn KL, Farrington MK, Creazzo T, Hawkins AF, Daskalakis N, Kwan SY, Ebersviller S, Burchette JL, et al. (2005) A mutation in the TRPC6 cation channel causes familial focal segmental glomerulosclerosis. Science 308:1801-1804.

Zhang W, Cheng LE, Kittelmann M, Li J, Petkovic M, Cheng T, Jin P, Guo Z, Göpfert MC, Jan LY, et al. (2015) Ankyrin repeats convey force to gate the NOMPC mechanotransduction channel. Cell 162:1391-1403.

Zhang Z, Tang J, Tikunova S, Johnson JD, Chen Z, Qin N, Dietrich A, Stefani E, Birnbaumer L, and Zhu MX (2001) Activation of Trp3 by inositol 1,4,5-trisphosphate receptors through displacement of inhibitory calmodulin from a common binding domain. Proc Natl Acad Sci USA 98:3168-3173.

Address correspondence to: Dr. Alexander Dietrich, Walther-StraubInstitute of Pharmacology and Toxicology, Nußbaumstr. 26, 80336 Munich, Germany. E-mail: Alexander.Dietrich@lrz.uni-muenchen.de 